\title{
Spectral Analysis of Polynomial Nonlinearity with Applications to RF Power Amplifiers
}

\author{
G. Tong Zhou \\ School of Electrical and Computer Engineering, Georgia Institute of Technology, Atlanta, GA 30332-0250, USA \\ Email:gtz@ece.gatech.edu \\ Raviv Raich \\ School of Electrical and Computer Engineering, Georgia Institute of Technology, Atlanta, GA 30332-0250, USA \\ Email: raviv@ece.gatech.edu
}

Received 1 September 2003; Revised 2 December 2003

The majority of the nonlinearity in a communication system is attributed to the power amplifier (PA) present at the final stage of the transmitter chain. In this paper, we consider Gaussian distributed input signals (such as OFDM), and PAs that can be modeled by memoryless or memory polynomials. We derive closed-form expressions of the PA output power spectral density, for an arbitrary nonlinear order, based on the so-called Leonov-Shiryaev formula. We then apply these results to answer practical questions such as the contribution of AM/PM conversion to spectral regrowth and the relationship between memory effects and spectral asymmetry.

Keywords and phrases: nonlinear, polynomial, power amplifier, spectral analysis.

\section{INTRODUCTION}

Power amplifiers (PAs) are important components of communications systems and are inherently nonlinear. For Example, the so-called class AB PAs, which are moderately nonlinear, are typically employed in wireless base stations and handsets. When a nonconstant modulus signal goes through a nonlinear PA, spectral regrowth (broadening) appears in the output, which in turn causes adjacent channel interference (ACI). Stringent limits on ACI are imposed by the standard bodies and thus the extent of the PA nonlinearity must be controlled.

We are interested in predicting the amount of spectral regrowth for a given level of PA nonlinearity. Since more linear PAs are less efficient, one may want to maximize nonlinearity (and hence optimize efficiency) subject to the spectral mask constraint. Such optimization strategy is feasible if we have tools for spectral regrowth analysis of the nonlinear output.

If the PA input is Gaussian, the PA output power spectral density (PSD) has been derived for a 5th-order nonlinear PA in $[1,2]$. In [3], the analysis was carried out for a 9th-order nonlinear PA. The results in [4] are fairly general but developed for bandpass signals, whereas references $[1,2,3]$ and the present paper adopt a baseband nonlinear formulation. In [5], a general expression is given without proof. When the PA input is non-Gaussian, theoretical analysis becomes more complicated, but results are available in [6] for a 7th-order nonlinear PA with (non-)Gaussian inputs.

The objective of this paper is to derive closed-form expressions for the PA output PSD (or output autocovariance function) for an arbitrary nonlinear order, for both the memoryless and memory baseband polynomial PA models. The PA input is assumed to be Gaussian distributed, which is a reasonable assumption for OFDM signals [2], forward link CDMA signals with a large number of Walsh-coded channels at the same frequency [7], or signals at the satellite-borne relay [4]. The Gaussian assumption significantly reduces the complexity of the analysis. Equipped with these formulas, we can then answer practical questions, such as how important or necessary it is to correct for the AM/PM distortion in the PA and possible mechanisms for spectral asymmetry in the PA output spectrum.

We would like to emphasize that the PA models considered in this paper belong to the polynomial family $[8,9]$; that is, polynomials or Taylor series for the (quasi) memoryless case, and Volterra series for the case with memory. Polynomials and Volterra series are frequently used in PA modeling; see, for example, $[1,2,3,4,6,9,10,11]$.

The organization of the paper is as follows. In Section 2, we outline the approach of spectral analysis for a baseband nonlinear system with cyclostationary input, suitable for digital communication signals. We will investigate the 
well-known (quasi) memoryless PA model in Section 3, and then study the relatively recent memory polynomial model in Section 4. Conclusions are drawn in Section 5. In order not to interrupt the flow of the paper, we defer the rather technical proofs of our theorems to Section 6.

\section{CYCLOSTATIONARY INPUT AND SPECTRAL ANALYSIS}

A digital communication signal $x(t)$ is represented by

$$
x(t)=\sum_{k} s_{k} h(t-k T),
$$

where $s_{k}$ is the $k$ th symbol, $h(t)$ is the pulse shaping filter, and $T$ is the symbol period. Thus, $x(t)$ is strict-sense cyclostationary in general [12, Chapter 12], [13].

We denote by $\operatorname{cum}\{\cdot\}$, the cumulant operator. The firstorder cumulant is the mean; the second-order cumulant is the covariance. General definitions and properties of cumulants can be found in [14]. The autocovariance function of the PA input signal $x(t)$ at time $t$ and lag $\tau$ is defined as

$$
c_{2 x}(t ; \tau)=\operatorname{cum}\left\{x^{*}(t), x(t+\tau)\right\} .
$$

Closed-form spectral analysis for a nonlinear system with nonstationary (or cyclostationary) input is in general extremely difficult (if at all possible), even under the Gaussian $x(t)$ assumption. Therefore, we focus our attention on the case where the bandwidth of the pulse shaping filter is limited to $1 / T$ (i.e., $h(t)$ has no excess bandwidth). Denote by $H(f)$ the Fourier transform (FT) of $h(t)$; that is,

$$
H(f)=\int h(t) e^{-j 2 \pi f t} d t
$$

this assumption implies that $H(f)=0$, for all $|f|>1 /(2 T)$.

If $s_{k}$ is zero mean, i.i.d. with variance $\sigma_{s}^{2}$, we show next that $x(t)$ in (1) is wide-sense stationary; that is, $c_{2 x}(t ; \tau)=$ $c_{2 x}(\tau)$, for all $t$.

First, it is straightforward to show that

$$
c_{2 x}(t ; \tau)=\sigma_{s}^{2} \sum_{k} h^{*}(t-k T) h(t+\tau-k T)
$$

for the $x(t)$ in (1). Next, recall the inverse FT relationship

$$
h(t)=\int H(f) e^{j 2 \pi f t} d f .
$$

Substituting (5) into (4) and using the fact that

$$
\sum_{m} \frac{1}{T} \delta\left(f-\frac{m}{T}\right)=\sum_{k} e^{j 2 \pi f k T}
$$

we obtain

$$
c_{2 x}(t ; \tau)=\frac{\sigma_{s}^{2}}{T} \sum_{m} e^{-j 2 \pi m t / T} \int H^{*}(f+m / T) H(f) e^{j 2 \pi f \tau} d f .
$$

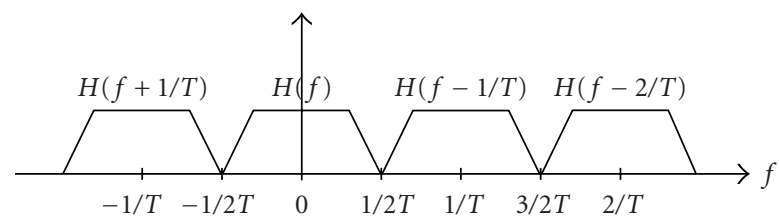

Figure 1: When $H(f)$ has no excess bandwidth, $H^{*}(f+m / T) H(f)$ $=0$, for all $m \neq 0$.

From (7), it is clear that the $t$-dependence in $c_{2 x}(t ; \tau)$ comes from the $e^{-j 2 \pi m t / T}$ term, if $m \neq 0$. Equation ( 7 ) can also be viewed as a synthesis equation for the time-varying correlation function in terms of cyclic correlation with cycles $-2 \pi m / T$. The bandwidth of $H(f)$ affects the number of cycles present in $c_{2 x}(t ; \tau)[15,16]$.

Since the bandwidth of $H(f)$ is limited to $1 / T, H(f+$ $m / T$ ) and $H(f)$ do not overlap if $m \neq 0$ (see Figure 1 ), and hence the product $H^{*}(f+m / T) H(f)=0$, for all $m \neq 0$. As a result, only the $m=0$ term survives in the summation in (7) and

$$
c_{2 x}(t ; \tau)=\frac{\sigma_{s}^{2}}{T} \int|H(f)|^{2} e^{j 2 \pi f \tau} d f,
$$

which is not a function of $t$. Therefore, under the no excess bandwidth assumption, $c_{2 x}(t ; \tau)=c_{2 x}(\tau)$, for all $t$, meaning that $x(t)$ is wide-sense stationary.

Since all cumulants of order $\geq 3$ vanish for Gaussian processes, a wide-sense stationarity Gaussian $x(t)$ is also strict-sense stationarity. From now on, we will drop the $t$ dependence and express the autocovariance function of $x(t)$ as $c_{2 x}(\tau)$.

We point out that (wide-sense) stationarity of $x(t)$ is assumed in $[1,2,3,4,6]$, often without justification.

The PSD of $x(t)$ is defined as the FT of $c_{2 x}(\tau)$ :

$$
S_{2 x}(f)=\int c_{2 x}(\tau) e^{-j 2 \pi f \tau} d \tau
$$

Next, we will relate the PSD of the baseband PA output $y(t)$ to that of the baseband PA input $x(t)$, when $x(t)$ and $y(t)$ obey polynomial nonlinear relationships.

\section{QUASIMEMORYLESS PA MODEL}

The following model is commonly used to describe memoryless PAs in the baseband; see, for example, [10, page 69],

$$
\begin{aligned}
y(t) & =\sum_{k=0}^{K} a_{2 k+1}[x(t)]^{k+1}\left[x^{*}(t)\right]^{k} \\
& =x(t) \sum_{k=0}^{K} a_{2 k+1}|x(t)|^{2 k},
\end{aligned}
$$

where $\left\{a_{2 k+1}\right\}$ are the (complex-valued) coefficients for the PA. We see from (11) that the complex gain is $G(x(t))=$ $y(t) / x(t)=\sum_{k=0}^{K} a_{2 k+1}|x(t)|^{2 k}$, which is a function of $r=$ $|x(t)|$ only. 
Writing the complex gain as $G(r)=A(r) e^{j \Phi(r)}$, we refer to $A(r)$ as the AM/AM conversion, and to $\Phi(r)$ as the $\mathrm{AM} / \mathrm{PM}$ conversion. A linear PA would have constant $A(r)$ and $\Phi(r)$ characteristics. If $A(r)$ is nonconstant but $\Phi(r)$ is, the corresponding PA is called strictly memoryless. If both $A(r)$ and $\Phi(r)$ are nonconstant, the resulting PA is called quasimemoryless. Equation (10) can be used to describe both types of memoryless nonlinearity, and hence we do not distinguish the two in subsequent analysis.

\subsection{Closed-form expression for spectral regrowth}

We assume that $x(t)$ is circular complex in the sense that

$$
\operatorname{cum}\{x(t), x(t+\tau)\}=0, \quad \forall \tau \text {. }
$$

We write $x(t)=x_{R}(t)+j x_{I}(t)$, where $x_{R}(t)$ and $x_{I}(t)$ are the real and imaginary parts of $x(t)$, respectively. It can be shown that (12) is equivalent to

$$
\begin{gathered}
\operatorname{cum}\left\{x_{R}(t), x_{R}(t+\tau)\right\}=\operatorname{cum}\left\{x_{I}(t), x_{I}(t+\tau)\right\}, \\
\operatorname{cum}\left\{x_{R}(t), x_{I}(t+\tau)\right\}=-\operatorname{cum}\left\{x_{I}(t), x_{R}(t+\tau)\right\} .
\end{gathered}
$$

Processes satisfying (12) have also been referred to as complex video processes [17]. This assumption is commonly used; see $[1,2,3,4,6]$.

We now present the first theorem which relates the output PSD $S_{2 y}(f)$ to the input PSD $S_{2 x}(f)$ and (quasi) memoryless PA parameters $\left\{a_{2 k+1}\right\}$.

Theorem 1. Assume that $x(t)$ is stationary, zero-mean, complex Gaussian distributed and satisfies (12). If the output $y(t)$ is related to the input $x(t)$ through (10), then the autocorrelation function of $y(t)$ is

$$
c_{2 y}(\tau)=\sum_{m=0}^{K} \alpha_{2 m+1}\left|c_{2 x}(\tau)\right|^{2 m} c_{2 x}(\tau)
$$

where the constant coefficient

$$
\begin{gathered}
\alpha_{2 m+1}=\frac{1}{m+1}\left|\sum_{k=m}^{K} a_{2 k+1}\left(\begin{array}{c}
k \\
m
\end{array}\right)(k+1) !\left[c_{2 x}(0)\right]^{k-m}\right|^{2}, \\
\left(\begin{array}{c}
k \\
m
\end{array}\right)=\frac{k !}{m !(k-m) !} .
\end{gathered}
$$

The PSD of $y(t)$ is related to that of $x(t)$ through

$$
\begin{aligned}
S_{2 y}(f)=\sum_{m=0}^{K} \alpha_{2 m+1} \underbrace{S_{2 x}(f) \star \cdots \star S_{2 x}(f)}_{m+1} \\
\star \underbrace{S_{2 x}(-f) \star \cdots \star S_{2 x}(-f)}_{m},
\end{aligned}
$$

where $\star$ denotes convolution.

Proof. See Section 6.1.
Some remarks are now in order.

(R1) From (16), we infer that if $S_{2 x}(f)$ has bandwidth $B_{x}$, $y(t)$ has bandwidth $B_{y}=(2 K+1) B_{x}$, due to the spectral expansion caused by the convolution.

(R2) If $S_{2 x}(f)$ is symmetric; that is, $S_{2 x}(f)=S_{2 x}(-f)$, then $S_{2 y}(f)$ is symmetric as well. This means that a (quasi) memoryless PA will not lead to spectral asymmetry in the PA output.

(R3) If $S_{2 x}(f)$ is asymmetric, the $2 m$ times spectral convolution on the RHS of (16) will yield a more symmetric spectrum for larger $m$.

Next, we would like to provide detailed expressions for the 9th-order nonlinear PA; that is, $K=4$ in (10). Equation (16) yields for $K=4$,

$$
\begin{gathered}
\alpha_{1}=\left|a_{1}+2 a_{3} c_{2 x}(0)+6 a_{5} c_{2 x}^{2}(0)+24 a_{7} c_{2 x}^{3}(0)+120 a_{9} c_{2 x}^{4}(0)\right|^{2} \\
\alpha_{3}=2\left|a_{3}+6 a_{5} c_{2 x}(0)+36 a_{7} c_{2 x}^{2}(0)+240 a_{9} c_{2 x}^{3}(0)\right|^{2} \\
\alpha_{5}=12\left|a_{5}+12 a_{7} c_{2 x}(0)+120 a_{9} c_{2 x}^{2}(0)\right|^{2} \\
\alpha_{7}=144\left|a_{7}+20 a_{9} c_{2 x}(0)\right|^{2} \\
\alpha_{9}=2880\left|a_{9}\right|^{2}
\end{gathered}
$$

It is important to cross-verify (17) with previously published results to validate our closed-form expression. We will compare with three references below.

(i) In [1], $c_{2 x}(\tau)$ was defined as $0.5 \operatorname{cum}\left\{x^{*}(t), x(t+\tau)\right\}[1$, equation (27)]. Once we have taken care of this scaling difference, (17) can be shown to agree with equation $(38)^{1}$ of [1], which holds for up to 5th-order nonlinearities.

(ii) In [6], $x(t)$ was assumed to be circular complex symmetric which renders $c_{2 x}(\tau)$ real valued. Except for the $\left[c_{2 x}(\tau)\right]^{2 m+1}$ vs. $\left|c_{2 x}(\tau)\right|^{2 m} c_{2 x}(\tau)$ difference, (17) agree with the expressions presented in [6, Section III.B], where a 7 th-order nonlinear model was considered.

(iii) In [3], the output PSD expression was obtained for a 9th-order nonlinear PA model. ${ }^{2}$ Our equations (17) agree with the expressions ${ }^{3}$ found on [3, page 1068].

In conclusion, previously published results in $[1,3,6]$ can be regarded as special cases of our closed-form expression (16).

\subsection{Case study: the effect of $A M / P M$ conversion on spectral regrowth}

Although by reducing the input power level to the PA (i.e., with input back-off), one can reduce the amount of spectral

\footnotetext{
${ }^{1}$ Reference [1] has a typo in equation (38): $48 \mathcal{R}\left\{\eta_{1} \eta_{3}^{*}\right\}$ should be $48 \mathcal{R}\left\{\eta_{1} \eta_{5}^{*}\right\}$.

${ }^{2}$ Although the baseband input-output relationship is incorrectly expressed in [3, equation (7)], the correct baseband model was used in [3, equation (A.5)].

${ }^{3}$ Reference [3] has a typo on page $1068: 15 \tilde{a}_{9} R_{z o}$ should be $20 \tilde{a}_{9} R_{z o}$.
} 


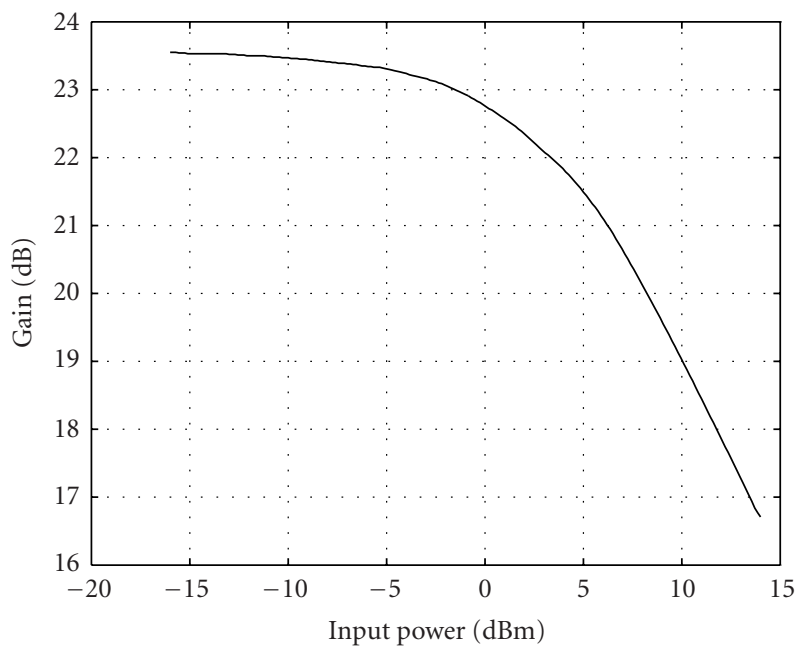

(a) AM/AM.

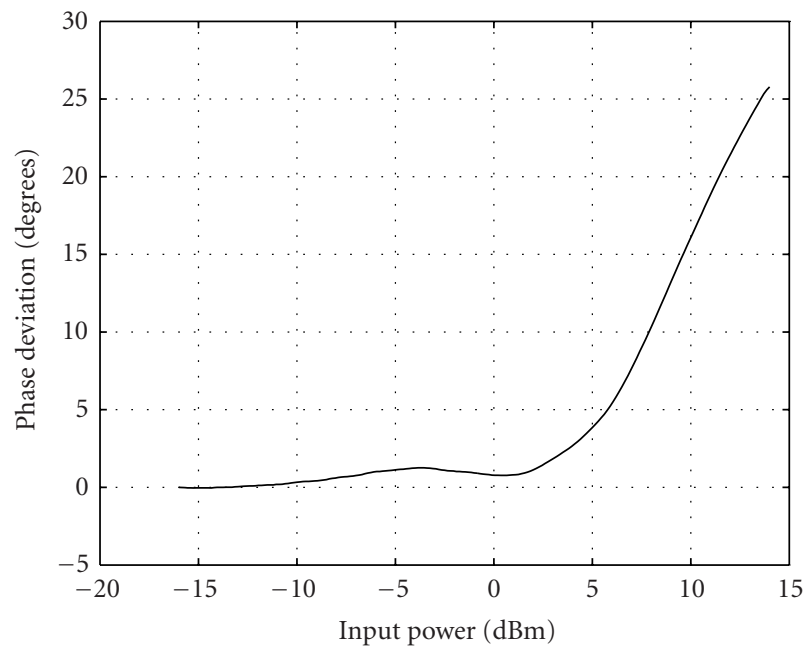

(b) AM/PM.

FIgure 2: Measured AM/AM and AM/PM characteristics of a Class AB PA.

TABLE 1: Estimated polynomial PA model coefficients for three scenarios: (i) when both AM/AM and AM/PM conversions are present; (ii) when only the AM/AM conversion is present $(\Phi(r)=0)$; and (iii) when only the AM/PM conversion is present $(A(r)=11.75$ was used).

\begin{tabular}{|c|c|c|c|}
\hline Scenarios & (i) $\mathrm{AM} / \mathrm{AM}+\mathrm{AM} / \mathrm{PM}$ & (ii) AM/AM only & (iii) AM/PM only \\
\hline$a_{1}$ & $14.8526-j 0.1337$ & 14.8469 & $11.7443-j 0.1562$ \\
\hline$a_{3}$ & $-23.1899+j 6.9785$ & -23.3505 & $0.4681+j 5.9639$ \\
\hline$a_{5}$ & $30.5226-j 1.9699$ & 33.8272 & $-4.7569+j 6.9758$ \\
\hline$a_{7}$ & $-21.5517-j 4.7097$ & -25.4177 & $4.8612-j 13.7023$ \\
\hline$a_{9}$ & $6.0311+j 2.7527$ & 7.3773 & $-1.5655+j 5.6319$ \\
\hline
\end{tabular}

regrowth, the efficiency of the PA is also diminished. Some form of PA linearization is often sought in order to achieve both good linearity and efficiency. In order to adopt an effective linearization strategy, it is important to understand the nonlinear effects present and their manifestation in terms of spectral regrowth. ${ }^{4}$ For a given (quasi) memoryless PA, it is useful to assess the relative contributions from the AM/AM and $\mathrm{AM} / \mathrm{PM}$ conversions to spectral regrowth. We can do so using Theorem 1.

Given measured PA AM/AM characteristic $A(r)$ and AM/PM characteristic $\Phi(r)$, we can then calculate the complex gain $G(r)=A(r) e^{j \Phi(r)}$. Note that although the PA output $y(t)$ is a nonlinear function of the PA input $x(t), y(t)$ is linear in the model coefficients $\left\{a_{2 k+1}\right\}$. Therefore, regressing $r G(r)$ with respect to the basis $\left\{r, r^{3}, \ldots, r^{2 K+1}\right\}$, we can estimate the model parameters $\left\{a_{2 k+1}\right\}$ via linear least squares. Afterwards, we apply Theorem 1 to calculate the output PSD $S_{2 y}(f)$.

To assess the individual contribution from the AM/AM conversion to $S_{2 y}(f)$, we set, ${ }^{5} \Phi(r)=0$ and find the $\left\{a_{2 k+1}\right\}$

\footnotetext{
${ }^{4}$ The error vector magnitude should also be reduced, which is not the subject of this paper.

${ }^{5}$ If we set $\Phi(r)=c$, the PSD $S_{2 y}(f)$ can be shown to be independent of the constant $c$.
}

coefficients corresponding to $G(r)=A(r)$. On the other hand, to evaluate the individual contribution of the AM/PM effect to spectral regrowth, we set $A(r)=A$ (the intended linear gain of the PA), and find the $\left\{a_{2 k+1}\right\}$ coefficients corresponding to $G(r)=A e^{j \Phi(r)}$ as described in the previous paragraph.

Example 1. Figure 2 shows the AM/AM and AM/PM characteristics of an actual Class AB PA. Table 1 lists the extracted PA model parameters for three scenarios: (i) when both AM/AM and AM/PM conversions are present; (ii) when only the AM/AM conversion is present $(\Phi(r)=0)$; and (iii) when only the AM/PM conversion is present $(A(r)=11.75$ was used so that the corresponding output power $c_{2 y}(0)$ remains the same as in case (i) and case (ii)).

First, we would like to verify that the closed-form expression (16) is accurate. We generated 65,536 samples of the PA input $x(t)$ by passing a zero-mean, i.i.d., circular complex Gaussian process, through a 48-tap lowpass filter; the variance of $x(t)$ was set to $\sigma_{x}^{2}=c_{2 x}(0)=0.32^{2}$. The PA output $y(t)$ was formed according to $y(t)=x(t) A(|x(t)|) e^{j \Phi(|x(t)|)}$. The sample and the theoretical $S_{2 x}(f)$ and $S_{2 y}(f)$ are shown in Figure 3. The sample and the theoretical PSDs are very close (the dashed line and the dotted line almost coincide; the solid line and the dashed-dotted line almost coincide), indicating that formula (16) is accurate. Note that we have 


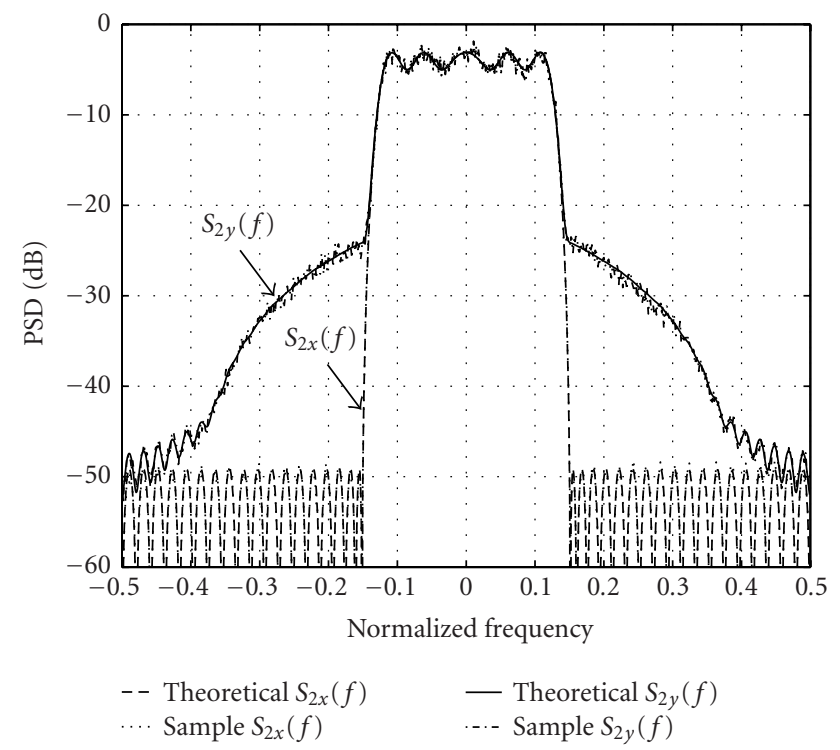

FIgURE 3: The theoretical $S_{2 x}(f)$ is shown by the dashed line, the sample $S_{2 x}(f)$ is shown by the dotted line; the theoretical $S_{2 y}(f)$ is shown by the solid line, and the sample $S_{2 y}(f)$ is shown by the dashed-dotted line.

lowered $S_{2 y}(f)$ by $21.4 \mathrm{~dB}$ to facilitate easier visual comparison between $S_{2 x}(f)$ and $S_{2 y}(f)$.

Next, we apply (16) to predict spectral regrowth for the above three scenarios. From Figure 4, we see that for the particular PA given in Figure 2 and for the Gaussian input described above, both AM/AM and AM/PM conversions contribute significantly to spectral regrowth. If one does not apply any linearization technique to the PA, the output PSD will be at the level indicated by the solid line in Figure 4. If with a linearization method, we can completely correct for the AM/AM distortion, the resulting $S_{2 y}(f)$ would be given by the dashed-dotted line, which is attributed solely to the AM/PM conversion. The remaining spectral regrowth is still high and additional linearization, aimed at reducing the AM/PM distortion, may be necessary.

In [18], a predistortion linearization algorithm was implemented for a handset which only corrects the AM/AM distortion of the PA. Example 1, however, shows that one should be careful not to underestimate the effects of AM/PM distortion. Of course, one has to evaluate the particular $A(r)$ and $\Phi(r)$ characteristics to draw pertinent conclusions.

\section{MEMORY POLYNOMIAL PA MODEL}

For low-power amplifiers and/or narrowband input, the PA can be regarded as (quasi) memoryless. However, highpower amplifiers (HPAs), such as those used in wireless base stations, exhibit memory effects; wideband signals (such as WCDMA) also tend to induce memory effects in the PA. In general, the cause of memory effects can be electrical

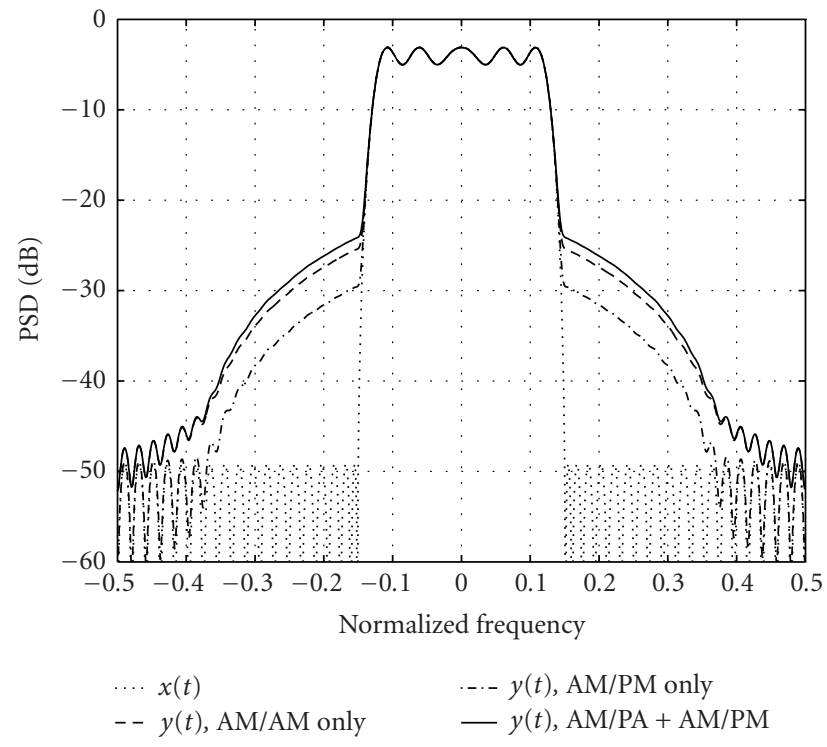

Figure 4: The theoretical $S_{2 x}(f)$ is shown by the dotted line, the theoretical $S_{2 y}(f)$ is shown by the solid line for scenario (i), by the dashed line for scenario (ii), and by the dashed-dotted line for scenario (iii).

or electrothermal [19]. When long-term memory effects are present, $\mathrm{AM} / \mathrm{AM}$ and $\mathrm{AM} / \mathrm{PM}$ conversions are insufficient to characterize the PA, and more elaborate models, such as the Volterra series, can be used; for example, $[9,20]$.

Although the Volterra series is a general nonlinear model with memory [8], its application to practical systems is limited due to the drastic increase in computational complexity when higher-order nonlinearities are included. Recently, in $[21,22]$, it has been shown that the so-called memory polynomial model is a good framework for studying nonlinear PAs with memory effects; it is also a good model for predistorters. When only odd-order nonlinear terms are considered, the PA output is related to the input as follows:

$$
\begin{aligned}
y(t) & =\sum_{k=0}^{K} \int h_{2 k+1}(\tau)|x(t-\tau)|^{2 k} x(t-\tau) d \tau \\
& =\sum_{k=0}^{K} \int h_{2 k+1}(\tau)[x(t-\tau)]^{k+1}\left[x^{*}(t-\tau)\right]^{k} d \tau \\
& =\sum_{k=0}^{K} \underbrace{h_{2 k+1}(t) \star \phi_{2 k+1}(x(t))}_{y_{2 k+1}(t)},
\end{aligned}
$$

where $\phi_{2 k+1}(x(t))=[x(t)]^{k+1}\left[x^{*}(t)\right]^{k}$.

To the best of our knowledge, there has been no published results on spectral regrowth analysis for nonlinear PAs with memory.

\subsection{Closed-form expression}

We present here a simple closed-form expression for the output PSD of the memory polynomial model (18). 
TABLE 2: Memory polynomial PA coefficients extracted for a real PA with maximum nonlinearity order $2 K+1=7$ and maximum lag $Q=2$.

\begin{tabular}{c|rrr}
\hline Diagonal kernel & $q=0$ & \multicolumn{1}{c}{$q=1$} & \multicolumn{1}{c}{\begin{tabular}{c} 
q \\
\hline$h_{1}[q]$
\end{tabular}} \\
$h_{3}[q]$ & $1.1330+j 0.0696$ & $-0.2027+j 0.0338$ & $0.0854-j 0.0341$ \\
$h_{5}[q]$ & $-0.2348-j 0.0876$ & $0.1809+j 0.2447$ & $-0.0439-j 0.0640$ \\
$h_{7}[q]$ & $0.2675-j 0.4113$ & $-0.1376-j 0.1862$ & $0.0888+j 0.0197$ \\
\hline
\end{tabular}

Theorem 2. Assume that $x(t)$ is stationary, zero-mean, complex Gaussian distributed and satisfies (12). If the output $y(t)$ is related to the input $x(t)$ through (18), then the PSD of $y(t)$ is related to that of $x(t)$ through

$$
\begin{array}{r}
S_{2 y}(f)=\sum_{m=0}^{K} \alpha_{2 m+1}(f) \underbrace{S_{2 x}(f) \star \cdots \star S_{2 x}(f)}_{m+1} \\
\star \underbrace{S_{2 x}(-f) \star \cdots \star S_{2 x}(-f)}_{m},
\end{array}
$$

where

$$
\begin{aligned}
& \alpha_{2 m+1}(f) \\
& =\frac{1}{m+1}\left|\sum_{k=m}^{K} H_{2 k+1}(f)\left(\begin{array}{c}
k \\
m
\end{array}\right)(k+1) !\left[c_{2 x}(0)\right]^{k-m}\right|^{2},
\end{aligned}
$$

and

$$
H_{2 k+1}(f)=\int h_{2 k+1}(t) e^{-j 2 \pi f t} d t
$$

is the FT of the $(2 k+1)$ th-order kernel $h_{2 k+1}(t)$.

Proof. See Section 6.2.

We have the following remarks.

(R4) The (quasi) memoryless model (10) can be regarded as a special case of the memory polynomial model (18) with $h_{2 k+1}(t)=a_{2 k+1} \delta(t)$. Therefore, Theorem 1 can be regarded as a special case of Theorem 2 with $H_{2 k+1}(f)=a_{2 k+1}$.

(R5) Since the baseband kernel $h_{2 k+1}(t)$ is generally complex valued, its FT is not guaranteed to be conjugate symmetric. Therefore, even if $S_{2 x}(f)$ is symmetric, $S_{2 y}(f)$ may not be symmetric.

\subsection{Case study: asymmetric spectral regrowth and memory effects}

It is commonly known that asymmetry in the PSD of $y(t)$ is indicative of memory effects in the PA (e.g., [11]). Since the memory polynomial model has been shown to be a good model for nonlinear PAs with memory, next, we will carry out quantitative analysis on spectral asymmetry of a PA with memory, by applying Theorem 2 . We use the adjacent chan- nel power ratio (ACPR) defined as [3]

$$
\operatorname{ACPR}=\frac{\int_{f_{3}}^{f_{4}} S_{2 y}(f) d f}{\int_{f_{1}}^{f_{2}} S_{2 y}(f) d f},
$$

as the performance metric, where $f_{1}$ and $f_{2}$ are the frequency limits of the main channel, and $f_{3}$ and $f_{4}$ are the frequency limits of the adjacent channel. The two bandwidths $\left(f_{2}-f_{1}\right)$ and $\left(f_{4}-f_{3}\right)$ need not be the same and indeed are not for many current standards [23, page 39]. For ACPR LOWER, we use $f_{3}, f_{4}$ as limits for the lower adjacent channel. Similarly,

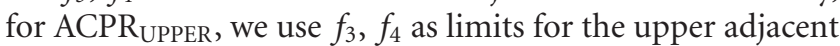
channel.

Example 2. In Table 2, we show the memory polynomial kernel coefficients extracted from a PA which is known to exhibit memory effects. The sampling rate was $f_{s}=150 \mathrm{MHz}$. To calculate the ACPR, we used $[-0.15,0.15]$ as the normalized frequency limits for the main channel, $[-0.45,-0.15]$ as the normalized frequency limits for the lower adjacent channel, and $[0.15,0.45]$ as the normalized frequency limits for the upper adjacent channel. In Figure 5, we plot ACPR $\mathrm{LOWER}$ as the solid line, and ACPR $\mathrm{UPPER}_{\text {as }}$ the dashed-dotted line, as a function of the input signal power $\sigma_{x}^{2}=c_{2 x}(0)$. The two curves do not coincide, implying spectral asymmetry in $S_{2 y}(f)$. At low input power levels, the ACPR curves are approximately constant-this is because the PA is approximately linear when it is largely backed off, and spectral regrowth was almost absent. As the PA is driven into compression, adjacent channel power increases sharply. Plots similar to Figure 5 can be used to select the input power level to ensure that spectral emission requirements are met.

\section{CONCLUSIONS}

The focus of this paper was on polynomial type of PA nonlinearities and Gaussian inputs. The objective was to obtain analytical expressions for the PA output power spectral density. We employed the little known Leonov-Shiryaev formula (see Section 6) to obtain closed-form output PSD expressions that apply to an arbitrary-order nonlinearity, and showed that they embody as special cases, previously reported results for memoryless nonlinear PAs of specific orders. Our spectral regrowth analysis on the PA model with memory is the first of its kind. These results can help us make important practical decisions such as what factors contribute to spectral regrowth and how to control or correct them in order to keep the adjacent channel interference to within limits. 


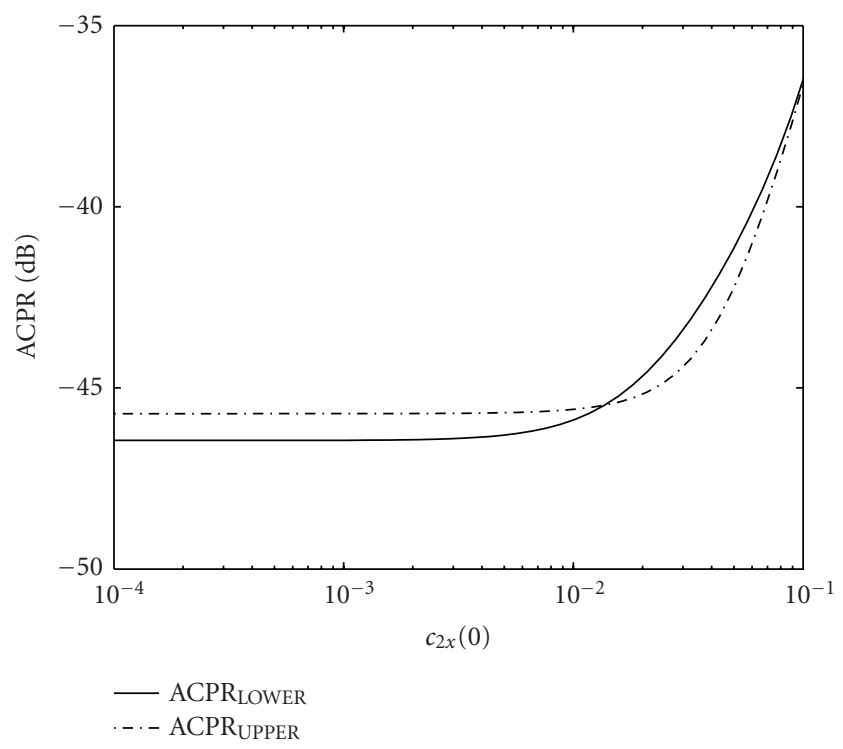

Figure 5: ACPR LOWER (solid line) and ACPR $_{\text {UPPER }}$ (dashed-dotted line) as a function of the input power $c_{2 x}(0)$ for a PA with memory.

\section{PROOFS OF THEOREMS}

\subsection{Proof of Theorem 1}

Define $\phi_{2 k+1}(x(t))=[x(t)]^{k+1}\left[x^{*}(t)\right]^{k}$. We can rewrite $(10)$ as

$$
y(t)=\sum_{k=0}^{K} a_{2 k+1} \phi_{2 k+1}(x(t)) .
$$

Since $x(t)$ is assumed to be zero-mean, Gaussian distributed, only the second-order statistics of $x(t)$ are nonzero. Moreover, all odd-order moments of $x(t)$ are zero [17]. Therefore, $E\left[\phi_{2 k+1}(x(t))\right]=0$ and $E[y(t)]=0$.

The autocorrelation (autocovariance) function of $y(t)$ is

$$
\begin{aligned}
c_{2 y}(\tau) & =\operatorname{cum}\left\{y^{*}(t), y(t+\tau)\right\} \\
& =\sum_{k=0}^{K} \sum_{l=0}^{K} a_{2 k+1}^{*} a_{2 l+1} \operatorname{cum}\left\{\phi_{2 k+1}^{*}(x(t)), \phi_{2 l+1}(x(t+\tau))\right\} .
\end{aligned}
$$

First, we would like to express $\operatorname{cum}\left\{\phi_{2 k+1}^{*}(x(t))\right.$, $\left.\phi_{2 l+1}(x(t+\tau))\right\}$ in terms of $c_{2 x}(\tau)$.

Since $\phi_{2 k+1}(x(t))$ is zero-mean,

$$
\begin{aligned}
\operatorname{cum} & \left\{\phi_{2 k+1}^{*}(x(t)), \phi_{2 l+1}(x(t+\tau))\right\} \\
= & E\left\{\left[x^{*}(t)\right]^{k+1}[x(t)]^{k}[x(t+\tau)]^{l+1}\left[x^{*}(t+\tau)\right]^{l}\right\} .
\end{aligned}
$$

It is possible to use the moment theorem for complex Gaussian processes [17] to simplify (28), but as the authors of [3] found out, it "requires overwhelmingly complex manual expansion of the moment expressions." We adopt another approach here, which employs the so-called Leonov-Shiryaev formula [14, page 89].
To utilize the Leonov-Shiryaev formula, we start with a two-way table. We list the individual elements that form the product $\phi_{2 k+1}^{*}(x(t))=\left[x^{*}(t)\right]^{k+1} x^{k}(t)$ in the first row and display the individual elements that form the product $\phi_{2 l+1}(x(t+\tau))=[x(t+\tau)]^{l+1}\left[x^{*}(t+\tau)\right]^{l}$ in the second row:

$$
\begin{gathered}
\underbrace{x^{*}(t) \cdots x^{*}(t)}_{k+1} \underbrace{x(t) \cdots x(t)}_{k} \\
\underbrace{x(t+\tau) \cdots x(t+\tau)}_{l+1} \underbrace{x^{*}(t+\tau) \cdots x^{*}(t+\tau)}_{l} .
\end{gathered}
$$

Next, we partition the above $(2 k+2 l+2)$ elements into subsets, according to the following criteria:

(i) the joint cumulant of the elements in any subset is nonzero,

(ii) for each partition, there must be at least one subset that contains elements from both rows of (29). We will refer to such subset as a "hooking" subset.

When both conditions (i) and (ii) are satisfied, the corresponding partition is called a "valid" partition. We must find all valid partitions of the two-way table in order to simplify (28).

Since $x(t)$ is zero-mean, Gaussian, and satisfies (12), the only nonzero cumulants of $x(t)$ are

$$
c_{2 x}(\tau)=\operatorname{cum}\left\{x^{*}(t), x(t+\tau)\right\}
$$

and its variants

$$
\begin{gathered}
c_{2 x}(0)=\operatorname{cum}\left\{x^{*}(t), x(t)\right\}, \\
c_{2 x}^{*}(\tau)=\operatorname{cum}\left\{x(t), x^{*}(t+\tau)\right\} .
\end{gathered}
$$

Therefore, to meet requirement (i), we only need to consider two element subsets, and the two elements within the subset must have different conjugation.

To illustrate the above concept, we consider the following two-way table which would be needed if we are interested in evaluating $\operatorname{cum}\left\{\phi_{5}^{*}(x(t)), \phi_{3}(x(t+\tau))\right\}$ :

$$
\begin{array}{ccccc}
x^{*}(t) & x^{*}(t) & x^{*}(t) & x(t) & x(t) \\
x(t+\tau) & x(t+\tau) & x^{*}(t+\tau) .
\end{array}
$$

One valid partition of the above 8 elements is

$$
\begin{aligned}
& \left\{x^{*}(t), x(t+\tau)\right\}, \quad\left\{x^{*}(t), x(t)\right\}, \quad\left\{x^{*}(t), x(t)\right\}, \\
& \left\{x(t+\tau), x^{*}(t+\tau)\right\},
\end{aligned}
$$

and there are 12 such possibilities (consider each element unique). In this partition, there is only one hooking subset $\left\{x^{*}(t), x(t+\tau)\right\}$.

Another valid partition is

$$
\begin{aligned}
& \left\{x^{*}(t), x(t+\tau)\right\}, \quad\left\{x^{*}(t), x(t+\tau)\right\}, \quad\left\{x(t), x^{*}(t+\tau)\right\}, \\
& \left\{x^{*}(t), x(t)\right\},
\end{aligned}
$$

and the multiplicity also happens to be 12 . In this partition, the first three subsets are hooking subsets. 
These are the only valid partitions for the above 8 element example.

Once we have found all valid partitions, we take the cumulant of the elements in each subset, multiply the resulting cumulants from all subsets of a given partition, and then sum over all valid partitions. For the above 8 element example, we have

$$
\begin{aligned}
\operatorname{cum}\{ & \left.\phi_{5}^{*}(x(t)), \phi_{3}(x(t+\tau))\right\} \\
= & 12 c_{2 x}(\tau) c_{2 x}(0) c_{2 x}(0) c_{2 x}(0) \\
& +12 c_{2 x}(\tau) c_{2 x}(\tau) c_{2 x}^{*}(\tau) c_{2 x}(0) \\
= & 12 c_{2 x}(\tau) c_{2 x}^{3}(0)+12\left|c_{2 x}(\tau)\right|^{2} c_{2 x}(\tau) c_{2 x}(0)
\end{aligned}
$$

Now for the general two-way table in (29), we realize the following. For each partition to be valid, there need to be $(2 m+1)$ hooking subsets: $(m+1)$ subsets are of the form $\left\{x^{*}(t), x(t+\tau)\right\}, m$ subsets are of the form $\left\{x(t), x^{*}(t+\tau)\right\}$, and $0 \leq m \leq \min (k, l)$. To come up with these $(2 m+1)$ hooking subsets, there are

$$
\begin{aligned}
\frac{(k+1) k \cdots(k+1-m)(l+1) l \cdots(l+1-m)}{(m+1) !} \\
\times \frac{k(k-1) \cdots(k-m+1) l(l-1) \cdots(l-m+1)}{m !}
\end{aligned}
$$

different possibilities.

Apart from the $(2 m+1)$ hooking subsets, the remaining elements must be grouped into $(k-m)$ subsets of the form $\left\{x^{*}(t), x(t)\right\}$, and $(l-m)$ subsets of the form $\left\{x(t+\tau), x^{*}(t+\right.$ $\tau)\}$. The multiplicity number for this stage is

$$
(k-m) !(l-m) !
$$

Multiplying (36) and (37), we find that the multiplicity number for a partition that involves exactly $(m+1)$ subsets of $\left\{x^{*}(t), x(t+\tau)\right\}, m$ subsets of $\left\{x(t), x^{*}(t+\tau)\right\},(k-m)$ subsets of $\left\{x^{*}(t), x(t)\right\}$, and $(l-m)$ subsets of $\left\{x(t+\tau), x^{*}(t+\tau)\right\}$ is

$$
\frac{1}{m+1}\left(\begin{array}{c}
k \\
m
\end{array}\right)\left(\begin{array}{c}
l \\
m
\end{array}\right)(k+1) !(l+1) !
$$

Now take the cumulant of each subset and multiply the resulting cumulants. We infer that the contribution from any partition described above to (28) is

$$
\left[c_{2 x}(\tau)\right]^{m+1}\left[c_{2 x}^{*}(\tau)\right]^{m}\left[c_{2 x}(0)\right]^{k-m}\left[c_{2 x}(0)\right]^{l-m} .
$$

Summing over all valid partitions, we obtain

$$
\begin{aligned}
\operatorname{cum}\left\{\phi_{2 k+1}^{*}(x(t)), \phi_{2 l+1}(x(t+\tau))\right\} & \\
=\sum_{m=0}^{\min (k, l)} & \frac{1}{m+1}\left(\begin{array}{c}
k \\
m
\end{array}\right)\left(\begin{array}{c}
l \\
m
\end{array}\right)(k+1) !(l+1) ! \\
& \quad \times\left|c_{2 x}(\tau)\right|^{2 m} c_{2 x}(\tau)\left[c_{2 x}(0)\right]^{k+l-2 m} .
\end{aligned}
$$

Substituting (40) into (27), we obtain

$$
\begin{aligned}
c_{2 y}(\tau)= & \sum_{k=0}^{K} \sum_{l=0}^{K} a_{2 k+1}^{*} a_{2 l+1} \sum_{m=0}^{\min (k, l)} \frac{1}{m+1}\left(\begin{array}{c}
k \\
m
\end{array}\right)\left(\begin{array}{c}
l \\
m
\end{array}\right) \\
& \times(k+1) !(l+1) !\left|c_{2 x}(\tau)\right|^{2 m} c_{2 x}(\tau)\left[c_{2 x}(0)\right]^{k+l-2 m} .
\end{aligned}
$$

The above equation can be simplified once we realize the following:

(i) $\sum_{k=0}^{K} \sum_{l=0}^{K} \sum_{m=0}^{\min (k, l)}$ is equivalent to $\sum_{m=0}^{K} \sum_{k=m}^{K} \sum_{l=m}^{K}$.

(ii) Since $c_{2 x}(0)=E\left[|x(t)|^{2}\right]$ is real-valued,

$$
\begin{aligned}
& \sum_{k=m}^{K} a_{2 k+1}^{*}\left(\begin{array}{c}
k \\
m
\end{array}\right)(k+1) !\left[c_{2 x}(0)\right]^{k-m} \\
&=\left[\sum_{l=m}^{K} a_{2 l+1}\left(\begin{array}{c}
l \\
m
\end{array}\right)(l+1) !\left[c_{2 x}(0)\right]^{l-m}\right]^{*} .
\end{aligned}
$$

Therefore,

$$
c_{2 y}(\tau)=\sum_{m=0}^{K} \alpha_{2 m+1}\left|c_{2 x}(\tau)\right|^{2 m} c_{2 x}(\tau)
$$

where

$$
\alpha_{2 m+1}=\frac{1}{m+1}\left|\sum_{k=m}^{K} a_{2 k+1}\left(\begin{array}{c}
k \\
m
\end{array}\right)(k+1) !\left[c_{2 x}(0)\right]^{k-m}\right|^{2} .
$$

Since the FT of $c_{2 x}(\tau)$ is $S_{2 x}(f)$, the FT of $c_{2 x}^{*}(\tau)$ is $S_{2 x}(-f)$. Thus, the input-output PSD relationship is given by (16).

\subsection{Proof of Theorem 2}

Define

$$
f_{k l}(\tau)=\int h_{k}^{*}(t) h_{l}(t+\tau) d t
$$

as the (deterministic) crosscorrelation function between the kernels $h_{k}(t)$ and $h_{l}(t)$.

Define

$$
g_{k l}(\tau)=\operatorname{cum}\left\{\phi_{k}^{*}(x(t)), \phi_{l}(x(t+\tau))\right\}
$$

as the (statistical) crosscorrelation function between $\phi_{k}(x(t))$ and $\phi_{l}(x(t))$. The expression for $g_{(2 k+1)(2 l+1)}(\tau)$ was found previously as (40).

From the linear systems theory, it is well known that if $y_{k}(t)=h_{k}(t) \star u_{k}(t), y_{l}(t)=h_{l}(t) \star u_{l}(t)$, then $\operatorname{cum}\left\{y_{k}^{*}(t), y_{l}(t+\tau)\right\}=f_{k l}(\tau) \star \operatorname{cum}\left\{u_{k}^{*}(t), u_{l}(t+\tau)\right\}$, where $f_{k l}(\tau)$ is given in $(45)$.

Since in the memory polynomial model $(20), y_{2 k+1}(t)=$ $h_{2 k+1}(t) \star \phi_{2 k+1}(x(t))$, we use our linear systems knowledge to infer

$$
c_{2 y}(\tau)=\sum_{k=0}^{K} \sum_{l=0}^{K} f_{(2 k+1)(2 l+1)}(\tau) \star g_{(2 k+1)(2 l+1)}(\tau) .
$$


Recall that the FT of $f_{k l}(\tau)$ is $H_{k}^{*}(f) H_{l}(f)$. Thus, the FT (47) yields

$$
S_{2 y}(f)=\sum_{k=0}^{K} \sum_{l=0}^{K} H_{2 k+1}^{*}(f) H_{2 l+1}(f) G_{(2 k+1)(2 l+1)}(f),
$$

where $G_{(2 k+1)(2 l+1)}(f)$ is the FT of $g_{(2 k+1)(2 l+1)}(\tau)$ given by (40).

Following the similar procedure as in Section 6.1, we can simplify $S_{2 y}(f)$ to $(21)-(22)$.

\section{ACKNOWLEDGMENTS}

The authors would like to thank Ning Chen for many insightful discussions on this paper. Appreciation also goes to Dr. J. S. Kenney for providing the PA measurements used in Figure 2. This work was supported in part by the National Science Foundation Grant ECS-0219262, the Georgia Electronic Design Center, and Danam USA Incorporated. Some results of this paper were presented at the EURASIP/IEEE Workshop on Nonlinear Signal and Image Processing, Trieste, Italy, June 2003.

\section{REFERENCES}

[1] S. P. Stapleton, G. S. Kandola, and J. K. Cavers, "Simulation and analysis of an adaptive predistorter utilizing a complex spectral convolution," IEEE Trans. Vehicular Technology, vol. 41, no. 4, pp. 387-394, 1992.

[2] N. Y. Ermolova, "Spectral analysis of nonlinear amplifier based on the complex gain Taylor series expansion," IEEE Communications Letters, vol. 5, no. 12, pp. 465-467, 2001.

[3] K. G. Gard, H. M. Gutierrez, and M. B. Steer, "Characterization of spectral regrowth in microwave amplifiers based on the nonlinear transformation of a complex Gaussian process," IEEE Trans. on Microwave Theory and Techniques, vol. 47, no. 7, pp. 1059-1069, 1999.

[4] N. Blachman, "The output signals and noise from a nonlinearity with amplitude-dependent phase shift," IEEE Trans. on Information Theory, vol. 25, no. 1, pp. 77-79, 1979.

[5] K. Gard, M. B. Steer, and L. E. Larson, "Generalized autocorrelation analysis of spectral regrowth from bandpass nonlinear circuits," in Proc. IEEE MTT-S International Microwave Symposium Digest, vol. 1, pp. 9-12, Phoenix, Ariz, USA, May 2001.

[6] G. T. Zhou and J. S. Kenney, "Predicting spectral regrowth of nonlinear power amplifiers," IEEE Trans. Communications, vol. 50, no. 5, pp. 718-722, 2002.

[7] V. Aparin, "Analysis of CDMA signal spectral regrowth and waveform quality," IEEE Trans. on Microwave Theory and Techniques, vol. 49, no. 12, pp. 2306-2314, 2001.

[8] V. J. Mathews and G. L. Sicuranza, Polynomial Signal Processing, John Wiley \& Sons, New York, NY, USA, 2000.

[9] S. A. Maas, Nonlinear Microwave Circuits, IEEE Press, Piscataway, NJ, USA, 1997.

[10] S. Benedetto and E. Biglieri, Principles of Digital Transmission with Wireless Applications, Kluwer Academic/Plenum Publishers, New York, NY, USA, 1999.

[11] S. C. Cripps, RF Power Amplifiers for Wireless Communications, Artech House, Norwood, Mass, USA, 1999.

[12] W. A. Gardner, Introduction to Random Processes with Applications to Signals and Systems, McGraw-Hill, New York, NY, USA, 2nd edition, 1990.
[13] G. B. Giannakis, "Cyclostationary signal analysis," in Digital Signal Processing Handbook, V. K. Madisetti and D. B. Williams, Eds., Chapter 17, CRC Press, Boca Raton, Fla, USA, 1998.

[14] D. R. Brillinger, Time Series: Data Analysis and Theory, Holden-Day, San Francisco, Calif, USA, 1981.

[15] C. M. Spooner and W. A. Gardner, "The cumulant theory of cyclostationary time-series. II. Development and applications," IEEE Trans. Signal Processing, vol. 42, no. 12, pp. 34093429, 1994.

[16] P. Ciblat, P. Loubaton, E. Serpedin, and G. B. Giannakis, "Asymptotic analysis of blind cyclic correlation-based symbol-rate estimators," IEEE Trans. on Information Theory, vol. 48, no. 7, pp. 1922-1934, 2002.

[17] I. Reed, "On a moment theorem for complex Gaussian processes," IEEE Trans. on Information Theory, vol. 8, no. 3, pp. 194-195, 1962.

[18] S. Kusunoki, K. Yamamoto, T. Hatsugai, et al., "Power amplifier module with digital adaptive predistortion for cellular phone," in Proc. IEEE MTT-S International Microwave Symposium Digest, vol. 2, pp. 765-768, Seattle, Wash, USA, June 2002.

[19] J. H. K. Vuolevi, T. Rahkonen, and J. P. A. Manninen, "Measurement technique for characterizing memory effects in RF power amplifiers," IEEE Trans. on Microwave Theory and Techniques, vol. 49, no. 8, pp. 1383-1389, 2001.

[20] W. Bosch and G. Gatti, "Measurement and simulation of memory effects in predistortion linearizers," IEEE Trans. on Microwave Theory and Techniques, vol. 37, no. 12, pp. 18851890, 1989.

[21] J. Kim and K. Konstantinou, "Digital predistortion of wideband signals based on power amplifier model with memory," Electronics Letters, vol. 37, no. 23, pp. 1417-1418, 2001.

[22] L. Ding, G. T. Zhou, D. R. Morgan, et al., "A robust digital baseband predistorter constructed using memory polynomials," IEEE Trans. Communications, vol. 52, no. 1, pp. 159-165, 2004.

[23] P. B. Kennington, High-Linearity RF Amplifier Design, Artech House, Norwood, Mass, USA, 2000.

G. Tong Zhou received her B.S. degree in biomedical engineering and instrumentation from the Tianjin University, China, in July 1989. From September 1989 to May 1995, she was with the University of Virginia (UVA), where she obtained her M.S. degree in biophysics in May 1992, her M.S. degree in electrical engineering in January 1993, and her Ph.D. degree in electrical engineering in January 1995. She was awarded

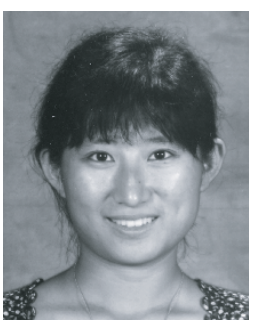
the 1995 Allan Talbott Gwathmey Memorial Award for outstanding research in the physical sciences at UVA, based on her Ph.D. dissertation. She has been with the School of Electrical and Computer Engineering at Georgia Institute of Technology since September 1995, and currently holds the rank of Associate Professor. In 1997, she received the National Science Foundation Faculty Early Career Development (CAREER) Award. She is also recipient of the 2000 Meritor Teaching Excellence Award at Georgia Institute of Technology. Dr. Zhou's research interests are in the general areas of statistical signal processing and communications. Specific current interests include predistortion linearization of nonlinear power amplifiers for wireless applications, communication channel identification and equalization, and bioinformatics. 
Raviv Raich was born in Israel. He received both the B.S. and M.S. degrees in electrical engineering from Tel-Aviv University, TelAviv, Israel, in 1994 and 1998, respectively. In 2004 he received the Ph.D. degree in electrical engineering from Georgia Institute of Technology, Atlanta, Georgia, USA. From 1994 to 1997, he served as an electronic engineer in the Israeli Defense Force. During 1998, he was with the Department of Elec-

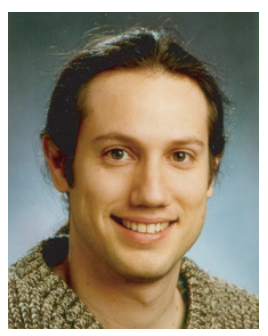

trical Engineering - Systems, Tel-Aviv University. During the same year, he was a consultant for Tadiran Electronic Systems, Ltd., Holon, Israel. During 1999 and 2000, he worked as a researcher with the communications team, Industrial Research Ltd., Wellington, New Zealand. His main research interests are predistortion linearization of nonlinear power amplifiers for wireless applications, statistical signal processing for communications, and estimation and detection theory. 